\title{
Strain-Tunable Half-Metallicity in Hybrid Graphene-hBN Monolayer Superlattices
}

\author{
Fanchao Meng ${ }^{1,(a), *, \text { Shiqi Zhang }}{ }^{2,(a)}$, In-Ho Lee ${ }^{3}$, Sukky Jun $^{4}$, and Cristian V. Ciobanu ${ }^{5, *}$
}

${ }^{1}$ Department of Mining and Materials Engineering, McGill University, Montréal, Québec H3A OC5, Canada

${ }^{2}$ School of Electrical, Computer and Energy Engineering, Arizona State University, Tempe, Arizona 85281, USA

${ }^{3}$ Korea Research Institute of Standards and Science, Daejeon 305-600, Korea

${ }^{4}$ Department of Mechanical Engineering, University of Wyoming, Laramie, Wyoming 82071, USA

${ }^{5}$ Department of Mechanical Engineering, Colorado School of Mines, Golden, Colorado 80401, USA

(a) These authors contributed equally to the work

*Corresponding authors, email: cciobanu@mines.edu; fanchao.meng@mail.mcgill.ca 


\begin{abstract}
As research in 2-D materials evolves towards combinations of different materials, interesting electronic and spintronic properties are revealed and may be exploited in future devices. A way to combine materials is the formation of spatially periodic domain boundaries in an atom-thick monolayer: as shown in recent reports, when these domains are made of graphene and hexagonal boron nitride, the resulting superlattice has half-metallic properties in which one spin component is (semi)metallic and the other is semiconductor. We explore here the range of spin-dependent electronic properties that such superlattices can develop for different type of domain boundaries, domain widths, and values of tensile strain applied to the monolayer. We show evidence of an interplay between strain and domain width in determining the electronic properties: while for armchair boundaries the bandgap is the same for both spin components, superlattices with zigzag boundaries exhibit rich spin-dependent behavior, including different bandgaps for each spin component, half-metallicity, and reversal of half-metallicity. These findings can lead to new ways of controlling the spintronic properties in hybrid-domain monolayers, which may be exploited in devices based on 2-D materials.
\end{abstract}

Keywords: 2-D materials; graphene; boron nitride; hybrid-domain; strain engineering; spintronics. 
Introduction. The isolation of graphene [1] and the rich physical phenomena [2-6] and properties [7-9] that it enjoys have sparked intense investigations, which have lately been directed towards large scale production $[10,11]$ and technological applications $[12,13]$. As graphene-related research spreads towards technological applications, fundamental experimental efforts are now increasingly addressing combinations of 2-D materials, such as monolayer hybrid domains [14-17] and layered heterostructures [18-22], which may offer access a finer range of desired electronic properties. As far as hybrid-domain monolayer are concerned, they exhibit very interesting properties such as the opening of a variable bandgap [23, 24], magnetism [25], unique thermal transport properties [26], robust half-metallic behavior without applied electric fields [27], and interesting interfacial electronic reconstructions [28]. Recent experiments aimed at creating graphene-hBN interfaces and hybrid domains graphene-hBN superlattices (CBNSLs) now enable access to some these properties [14-17].

Theoretical reports based on density functional theory (DFT) calculations have addressed some of the structural, electronic, and magnetic properties of CBNSLs. In particular, work by Pruneda [27] has elucidated the origin of half-metallicity in zigzag CBNSLs, showing that the magnetism of edge states leads to electronic reconstructions at the graphene-BN domain boundaries and gives rise to a band gap in only one of the spin components. The half-metallic property (i.e., the bandgap in one spin component and its absence in the other) can be controlled by the width of graphene and hBN domains, as long as they have zigzag interfaces [27, 29]. The interplay between mechanical and spin-dependent electronic properties has only recently been investigated [30]. Martinez-Gordillo and Pruneda have recently shown [30] that the application of electric fields to hybrid-domain superlattices with zigzag boundaries induces a mechanical deformation and a magnetic response; these authors also reported that mechanical stress could be used to control the magnetization of the domain boundaries and also induce half-metallicity.

Here, we analyze in detail the electronic properties of armchair and zigzag graphene-BN superlattices, subjected to strain parallel and perpendicular to the domain boundaries. For 
armchair boundaries, there is no electronic asymmetry between the two spin components, as the bandgap is the same for both; we show that the effect of strain is more pronounced when tension is applied perpendicular to the domain boundaries (as opposed to parallel) and identify the range of bandgap values that can be achieved by modifying the domain widths and the strain. For zigzag boundaries, we have found that while most zCBNSLs are half-metallic at zero strain, the application of strain leads to a rich set of electronic and spintronic properties beyond halfmetallicity: these include semiconductor behavior in each spin component with different bandgaps, semiconductor behavior in one spin component and semimetal in the other, and reversal of half-metallicity, i.e., the metallic spin component becomes semiconductor and vice versa. These properties can lead to new ways of controlling the spintronic properties in hybriddomain monolayers, which may be exploited in next generation devices based on 2-D materials. 
Computational Details. In this work, we have considered CBNSL with two types of boundaries, zigzag and armchair, which are the most common orientations observed experimentally [15]. We indicate the type of boundary for a given superlattice by the first letter in the notation, e.g., aCBNSL are superlattices with armchair boundaries. The notation follows the usual convention [24] for the supercell width, which is given by the number of zigzag chains for zCBNSL, and by the number of dimer lines across the width for aCBNSL. For example, Fig. 1 shows the zCBNSL4 and aCBNSL8 hybrid domain structures with widths of 4 zigzag lines [Fig. 1(a)] and 8 dimers [Fig. 1(b)], respectively.
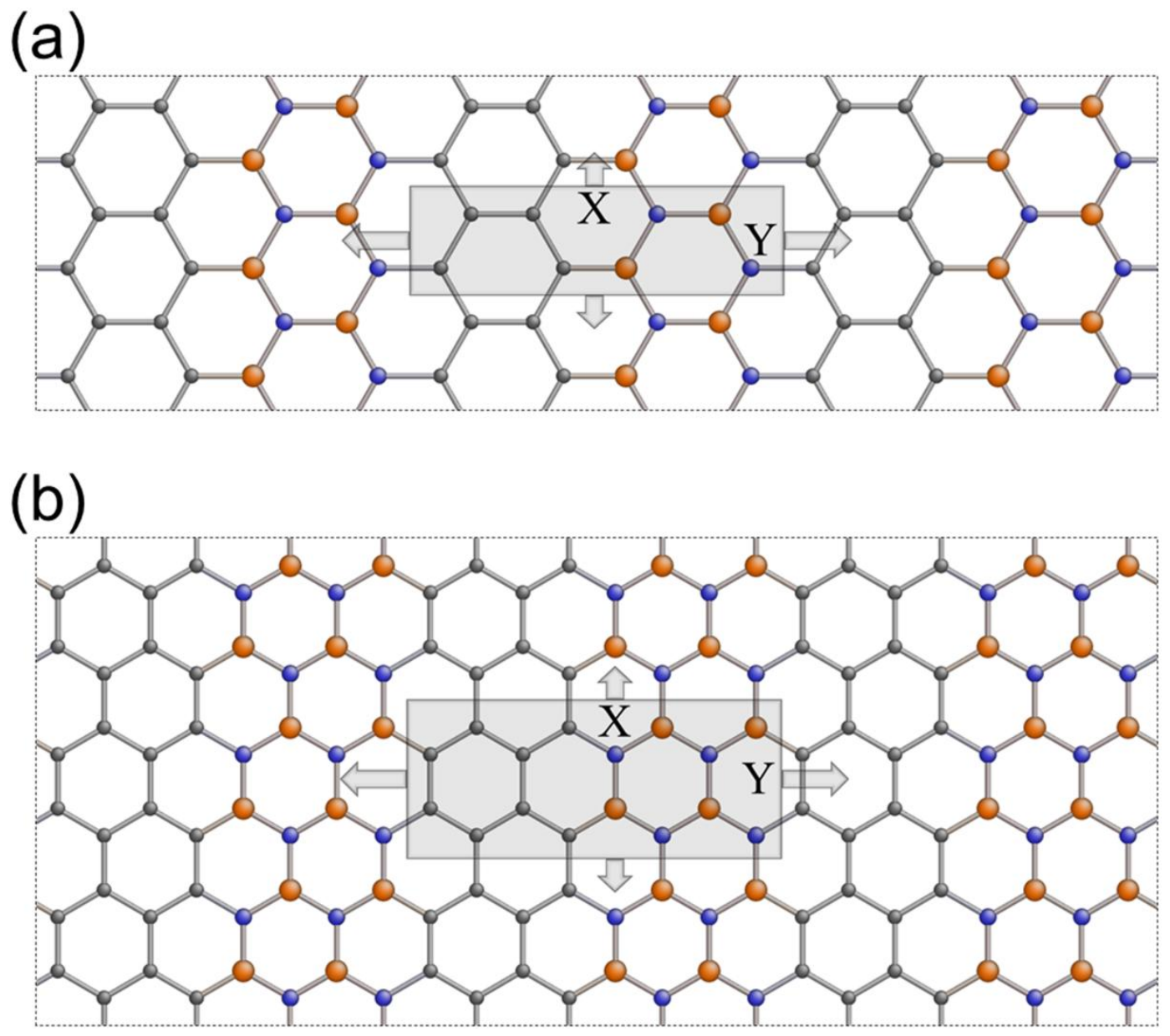

Figure 1. Hybrid-domain monolayer graphene-boron nitride superlattices (CBNSL) with (a) zigzag and (b) armchair boundaries. The specific structures are zCBNSL4 and aCBSNL8, for panels (a) and (b), respectively. Orange, blue, and gray represent boron, nitrogen, and carbon atoms, respectively. The shaded areas show the computational cells subjected to periodic boundary conditions, strained either parallel $(\mathrm{X})$ or perpendicular $(\mathrm{Y})$ to the direction of the domain boundaries. 
Superlattices with equally wide domains of graphene and hBN have been subjected to uniaxial tensile strains (along domain boundaries and perpendicular to them) ranging up to $19 \%$, a value which has been chosen towards the lower end of the range of recent theoretical reports on the limits within which graphene is still stable [31-36]. Given the very large number of calculations (strains in two directions, in increments of one percent, for multiple domain widths), for selected calculations of CBNSLs that are subjected to the same strain we use the Poisson effect to pre-compute the atomic positions. This is possible because even though the Poisson's ratio changes with the value and direction of the applied strain, it does not vary significantly with the width of the domains (Table I); in addition, we have performed spot-checks to ensure that the electronic properties are not unduly undermined by this efficient way to manage hundreds of calculations of deformed superlattices.

Table I. Comparison of Poisson's ratios for CBNSLs of different widths under same uniaxial strain

\begin{tabular}{|c|c|c|c|c|}
\hline \multirow{2}{*}{ aCBNSL } & \multicolumn{2}{|c|}{ Poisson's ratio } & \multirow{2}{*}{ zCBNSL } & \multirow{2}{*}{$\begin{array}{c}\text { Poisson's ratio } \\
10 \% \text { perpendicular }\end{array}$} \\
\hline & $15 \%$ perpendicular & $15 \%$ parallel & & \\
\hline 8 & 0.196833 & 0.111092 & 10 & 0.201666 \\
\hline 24 & 0.198971 & 0.115219 & 16 & 0.202369 \\
\hline 40 & 0.201358 & 0.117186 & 22 & 0.203856 \\
\hline
\end{tabular}

We have performed both structural optimization and spin-polarized electronic structure calculations using the SIESTA package [37], with Troulier-Martins pseudopotentials [38], and double- $\zeta$ plus polarization functions. For comparisons of trends, the structural relaxations were performed both in the local density approximation (LDA) and in the generalized gradient approximation (GGA). All electronic structures were computed in the GGA framework, with the Perdew-Burke-Ernzerkof functional [39]. The energy cut-off was set at 250 Ry [300 Ry for density of states calculations (DOS)], vacuum spacing in the direction perpendicular to the plane of the superlattice was set to $15 \AA$, and residual forces in conjugate-gradient structural 
relaxations were smaller than $0.03 \mathrm{eV} / \AA$ ( $0.01 \mathrm{eV} / \AA$ for DOS calculations $)$. We have determined the lattice constants for single-domain monolayer graphene $(2.463 \AA$ from LDA and $2.486 \AA$ from GGA) and BN (2.491 $\AA$ from LDA and $2.514 \AA$ from GGA), and have found structural properties similar to previous works $[24,40]$. We now describe our results regarding the effect of strain and domain size on the electronic properties of the superlattices. 
Results. First, our simulations show that CBNSLs with armchair domain boundaries do not exhibit asymmetry between the two spin components, and their bandstructure leads to the same bandgap for both spin components. This has been reported for unstrained superlattices [24], and we find that it holds true in the presence of strain as well. Figure 2 shows the variation of the bandgap with the periodic length of the superlattice (i.e., twice the width of a domain) for aCBNSLs subjected to strain perpendicular to the direction of the domain boundaries. The periodic lengths on the horizontal axis in Fig. 2 incorporate both the intrinsic width and the effect of the applied strain. The same aCBNSL intrinsic width (measured in dimers) is represented by points of the same color, and ranges from 12 to 48 dimers; the different points of the same color represent different levels of applied strain, at same intrinsic width. The application of tensile strain perpendicular to the domain boundary means that the width of the domains is directly modified (stretched), to the point that straining one intrinsic width by a sufficient value has approximately the same effect as increasing the intrinsic domain width without applying strain. This is suggested by the dash-line in Fig. 2, which joins the points in the graph in the order of their increasing (total or effective) supercell width. So, even though the perpendicular strain and the intrinsic widths are varied independently for our calculations of aCBNSLs, it appears that the total supercell width is the one parameter responsible for the (semi-quantitative) control of over the bandgap. 


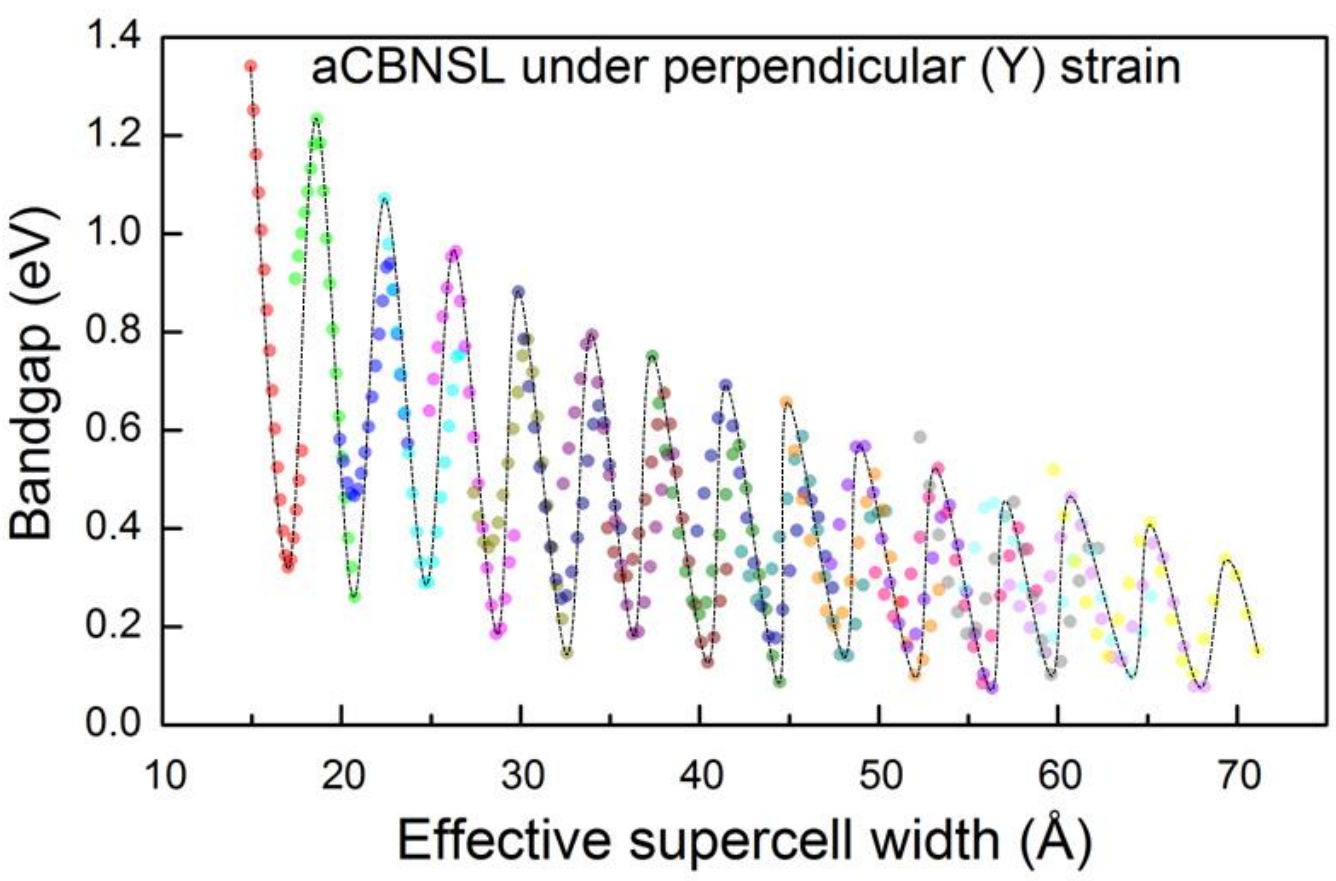

Figure 2. Bandgap of superlattices with armchair boundaries, subjected to perpendicular tensile strain. Points of same intrinsic width (measured in dimer lines) but with different strain values have the same color. The approximate oscillatory pattern indicates that the actual domain width (i.e., after being strained) is the relevant control parameter for the bandgap of aCBNSLs.

The aCBNSLs are semiconductor in nature with bandgap values ranging up to $\sim 1.35 \mathrm{eV}$, and with two main features apparent from Fig. 2: (i) there is a qualitative oscillatory pattern of the bandgap as a function of total domain width, and (ii) the amplitude of the bandgap oscillations decreases with increasing domain width. As the domain widths increase, the bandgap decreases in inverse proportion due to quantum confinement effects. The former feature, i.e. the oscillatory pattern, can be understood based on the pioneering results for graphene nanoribbons [41], which showed a three-family variation of the bandgap as a function of the width of the graphene ribbons. For aCBNSLs, the $\mathrm{C}$ atoms at the domain boundaries are bonded with $\mathrm{B}$ or $\mathrm{N}$ atoms (instead of $\mathrm{H}$ atoms in the case of nanoribbons), so the three-fold pattern variation as a function of the intrinsic domain width will still hold. In addition, we find that the strain provides a way to continuously change the width of the stripes, therefore leading to the definite 
oscillations shown in Fig. 2 (as opposed to the three-fold pattern observed in the case of graphene nanoribbons).

By comparison, when applying strains parallel to the domain boundaries, the intrinsic domain width and the strain remain two independent controls over the bandgap, with some overlap of the bandgap ranges achieved for neighboring intrinsic widths (Fig. 3). For the smallest width, the bandgap ranges from $\sim 1.1$ to $1.5 \mathrm{eV}$, whereas for the largest width studied the bandgap is between $\sim 0.2$ and $0.5 \mathrm{eV}$. The main difference with respect to perpendicular strains is that the parallel strain does not significantly alter the domain widths, owing to a Poisson ratio of only 0.11 (separate calculations, with some of the results listed in Table I): this small Poisson effect leads to much less coupling between the effects of strain and the intrinsic width (Fig. 3) as compared to the results described for perpendicular strain. In the remainder of the paper, we will only present the results of applying tensile strains perpendicular to the domain boundaries.

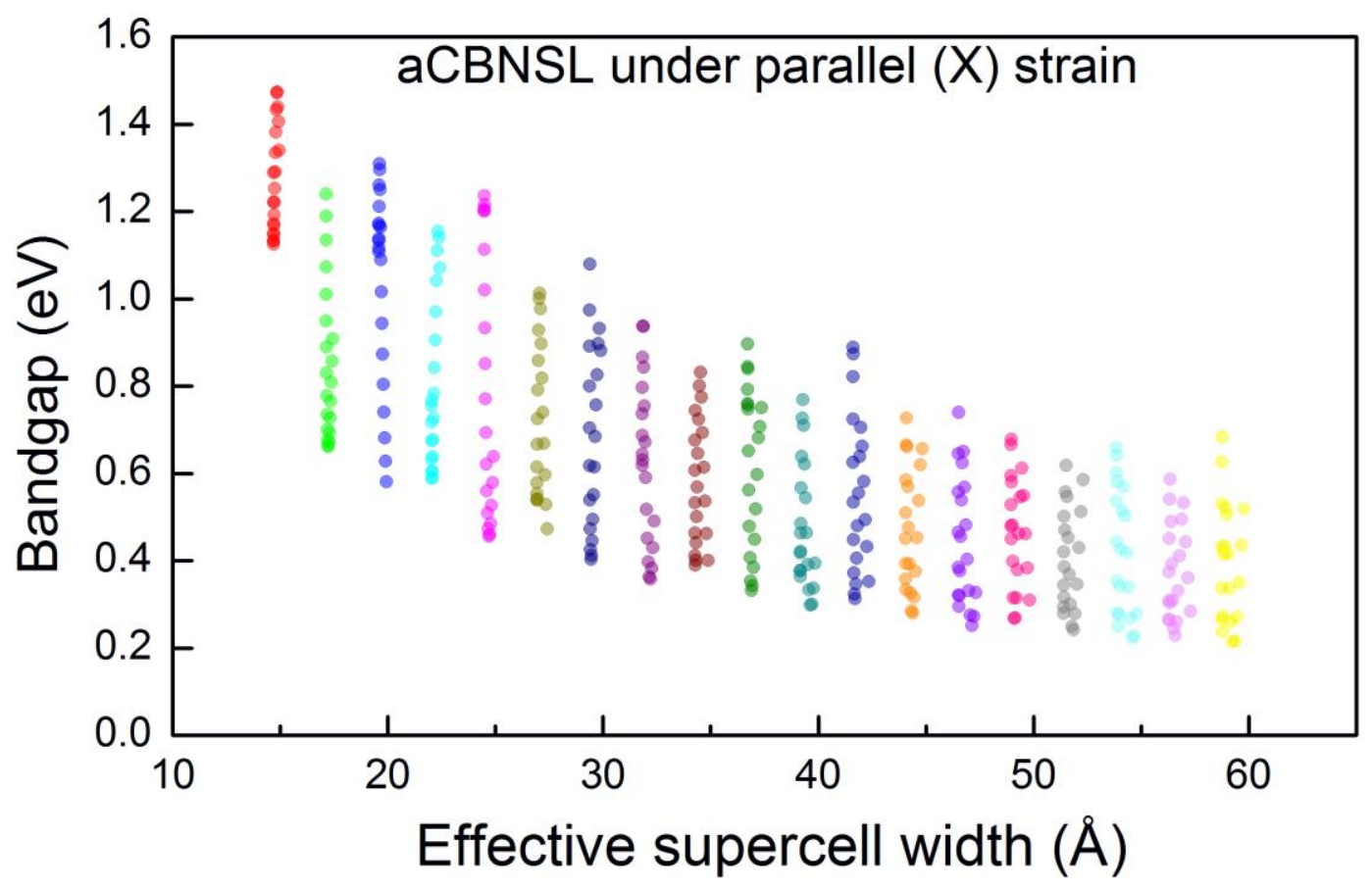

Figure 3. Bandgap of superlattices with armchair boundaries subjected to parallel tensile strain. Points of same intrinsic width (measured in dimer lines) but with different strain values have the same color. 
Focusing now on the CBNSLs with zigzag boundaries, our calculations in the absence of the strain display half-metallic behavior for zCBNSLs, in which one spin component shows a direct bandgap, and the other one is metallic. Figure 4(a) shows half-metallicity for zCBNSL with 10,18 , and 22 zigzag chains, with the bandgap of the spin-down component [blue line in Fig. 4(a)] decreasing with domain width. The half-metallic behavior is summarized in Fig. 4(b), where we plot the width-dependent bandgap for each spin component, and compare with unpolarized calculations.

(a)

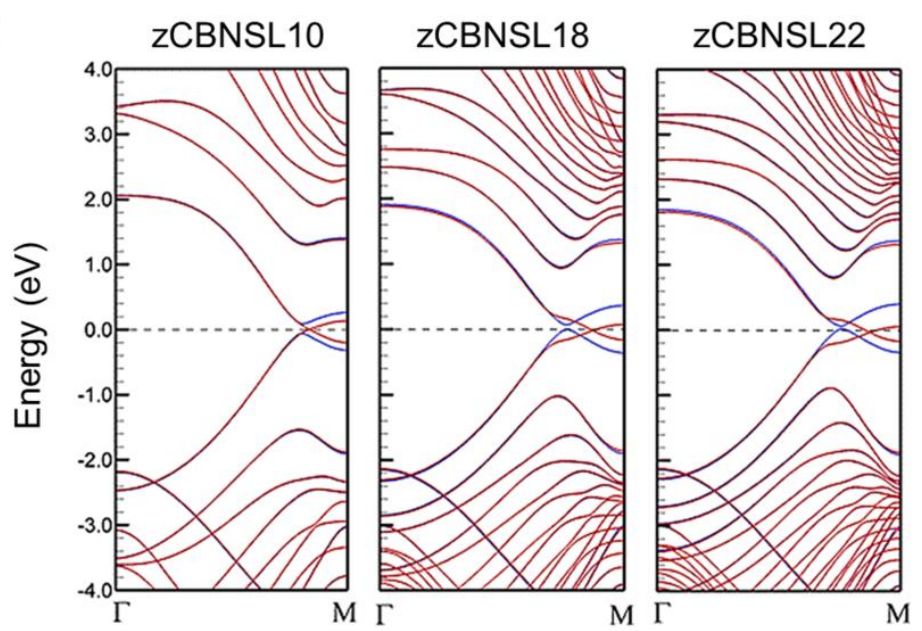

(b)

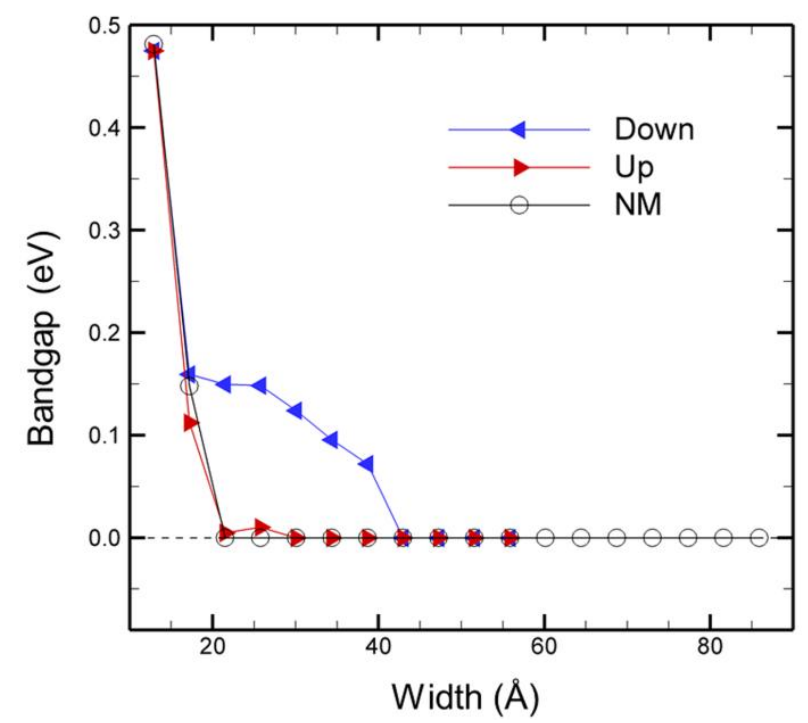

Figure 4. (a) Bandstructures of zCBNSL for three different supercell widths in the absence of strain, showing metallic behavior in the spin-up component (red) and semiconductor behavior for the spin-down component (blue). (b) Variation of bandgaps with the supercell width for zCBNSL; results of unpolarized (non-magnetic, NM) calculations are also shown for comparison. 
These results for zCBNSLs are consistent with other reports [27]. In addition, we report here the effect of strain in zCBNSLs. Two results representative of the effects of strain on the electronic properties of zigzag superlattices are shown in Fig. 5, for the zCBNSL10 and zCBNSL20 structures. For zCBNSL10, the application of increasing levels of strain increases the bandgap in the spin-down component, while the spin-up component remains metallic up to strains of about $7 \%$. Furthermore, at sufficiently large strain values, a bandgap opens in the (previously metallic) spin-up component, Fig. 5(a). Past $\sim 10 \%$ strain, the CBNSL is semiconducting in both spin components, but has the bandgaps are different.

A different manifestation of the combined effect of the domain width and applied strain can also be seen, for zCBNSL20 [Fig. 5(b)]. At low levels of strain, the spin-up component is metallic and spin-down is semiconducting; as the strain is increased, the metallic spin component becomes semiconducting and vice-versa [Fig. 5(b)]. 
(a)

zCBNSL10
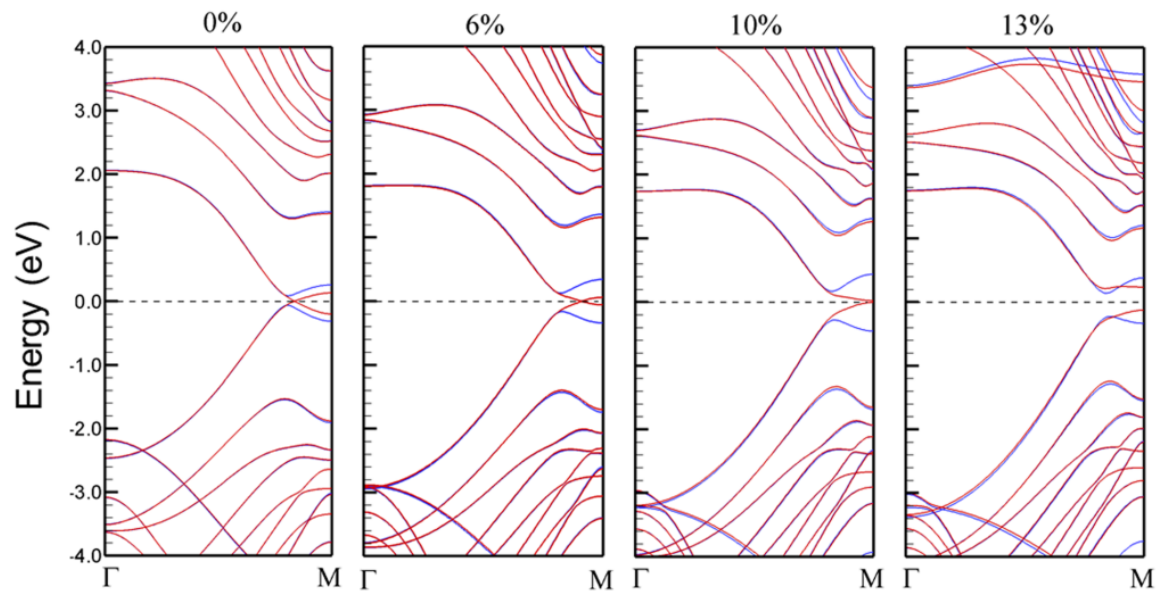

(b)

zCBNSL20
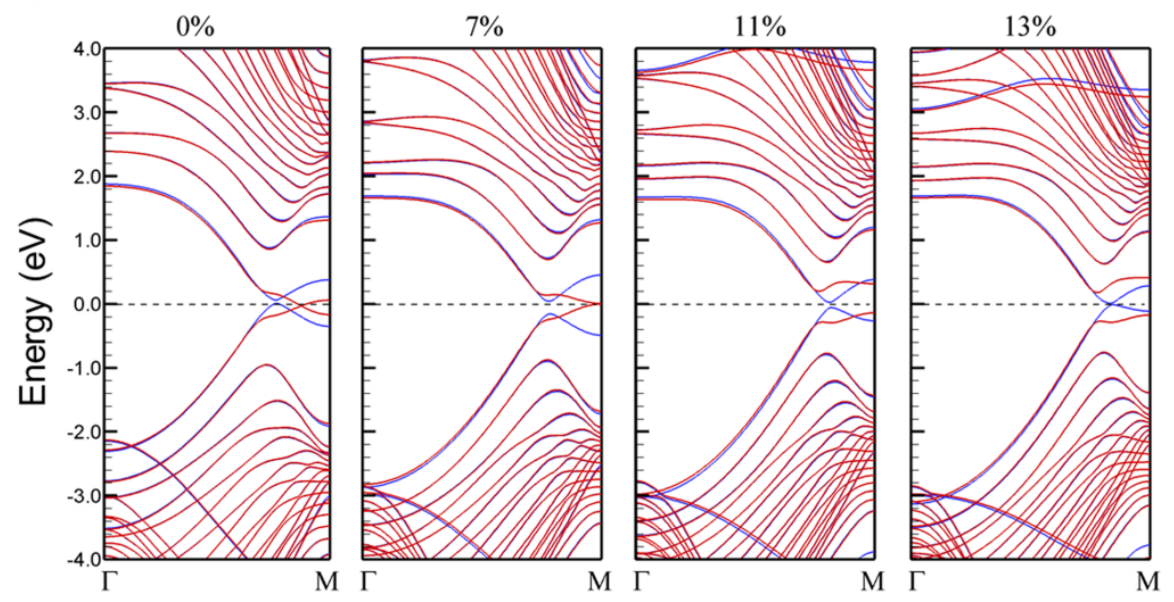

Figure 5. The effect of strain applied perpendicular to domain boundary for (a) zCBNSL10 and (b) zCBNSL20. The blue and red colors show the spin-down and spin-up components, respectively.

For zCBNSL structures with 10 to 22 zigzag lines, the spin-dependent bandgaps are plotted in Fig. 6 as a function of applied strain. One spin component (spin-up) is metallic up to about $7 \%$ level of strain, after which it develops a direct bandgap that increases monotonically with strain and starts to plateaus at different values for different domain widths [Fig. 6(a)]. The other spin component (spin-down), is semiconductor in the absence of strains for supercell widths smaller than 20 zigzag lines, and metallic for CBNSLs with supercells wider than 20 zigzag lines. As seen in Fig. 6(b), the bandgap for the spin-down component increases initially with strain, and then decreases past a value between $8 \%$ and $11 \%$ (depending on the width); it 
can in fact decrease to zero for sufficiently large domain widths (e.g., 24 zigzag chains or more).

(a)

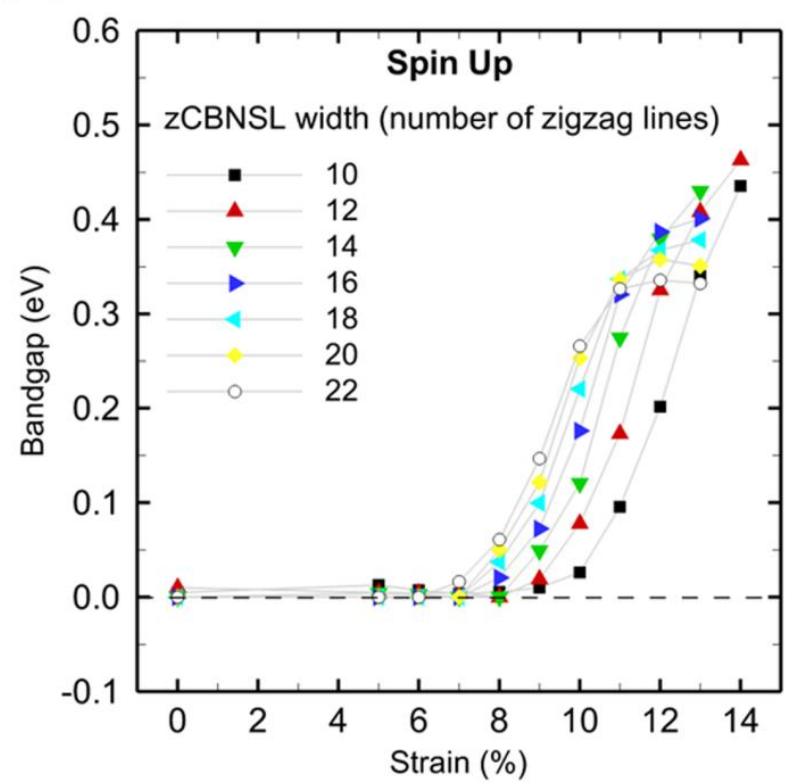

(b)

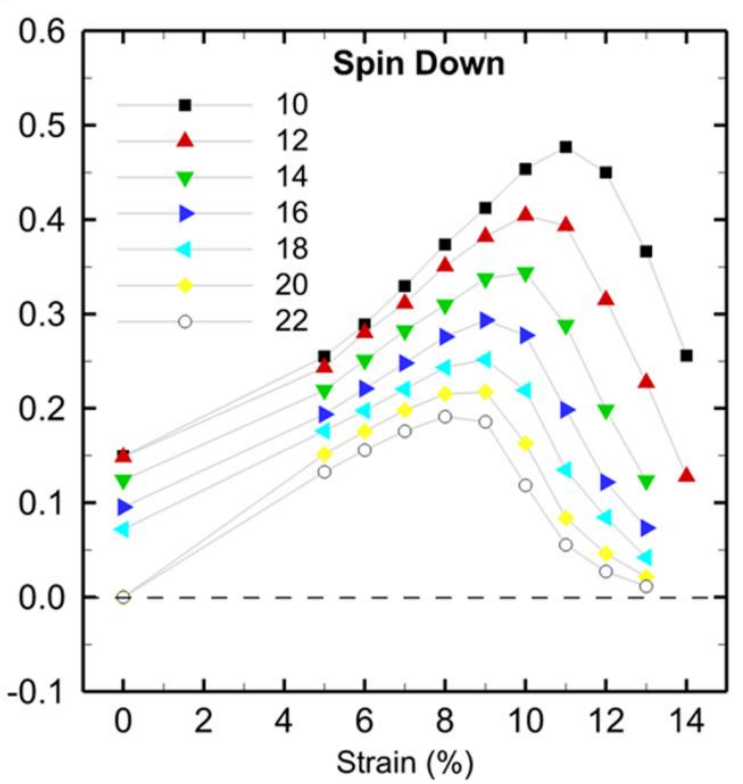

Figure 6. Bandgap for each spin component as a function of applied strain for zCBNSL of different intrinsic widths.

Discussion. In order to understand the underlying mechanism of the strain effects on the bandstructure of the two spin components, we plot the projected density of states (PDOS) of the $2 p$ orbitals for different strains perpendicular to the domain boundary (Fig. 7), using zCBNSL10 as a representative structure. Positive (negative) PDOS values represent the spin-up (spin-down). At $0 \%$ strain, the gap is (nearly) zero for the spin-up component, and $0.15 \mathrm{eV}$ for spin-down. With increasing strain, a gap $(\sim 0.03 \mathrm{eV})$ opens in the spin-up component at $10 \%$ strain and reaches $\sim 0.34 \mathrm{eV}$ at $13 \%$; the gaps measured from DOS and PDOS calculations are consistent with those in Fig. 6 obtained from bandstructure calculations. Interestingly, for low values of strain, below a $7 \%$ threshold, the $p$ states of $\mathrm{C}, \mathrm{B}$, and $\mathrm{N}$ are mixed or hybridized despite the physical separation between the $\mathrm{CB}$ and $\mathrm{CN}$ interfaces in the zCBNSLs. This means that there are PDOS peaks associated with the spin-up $p$ states of all three types of atoms at the same 
energy values [Fig. 7(a,b)]. Beyond that strain threshold [Fig. 7(c)], the hybridization largely separates in pairs of atom types so that the $\mathrm{C}$ and $\mathrm{N}$ states at the interfaces hybridize separately from the $\mathrm{C}$ and $\mathrm{B}$ states: therefore, now strain acts so as to decouple to a large extent the electronic interactions between the interfaces [Fig. 7(c)]. To our knowledge, this finding has not been reported previously and it cannot be gleaned from bandstructure calculations such as those Figs. 4 and 5. As the strain levels increase even further, the hybridization between the C, B, N states re-appears as a very complex mixing of the $\mathrm{C}, \mathrm{B}$, and $\mathrm{N}$ contributions [Fig. 7(d)]. Given that in the absence of strain half-metallicity is driven by the magnetism of interface states and electronic reconstructions [27], these re-hybridizations (Fig. 7) of the states at domain boundaries are very likely driving all the effects of strain on the spin-dependent bandstructures shown in Figs. 4, 5, and 6. 
(a) $0 \%$

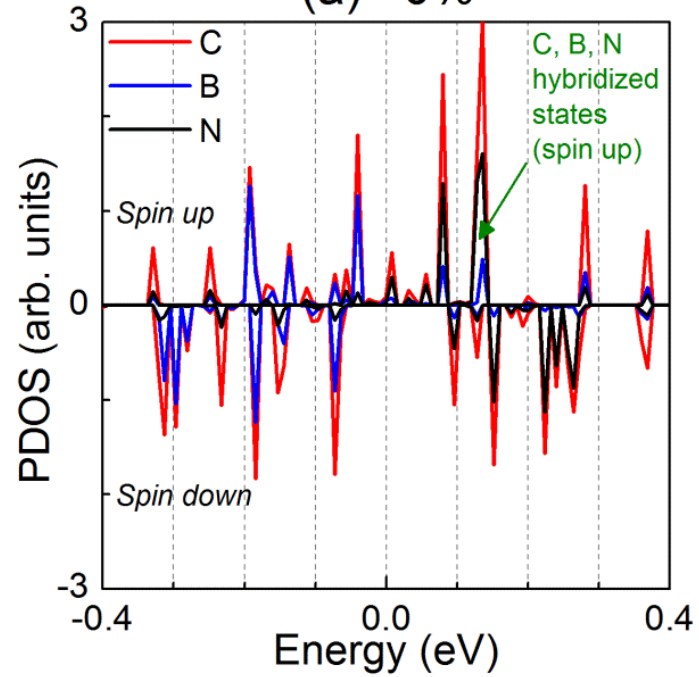

(c) $10 \%$

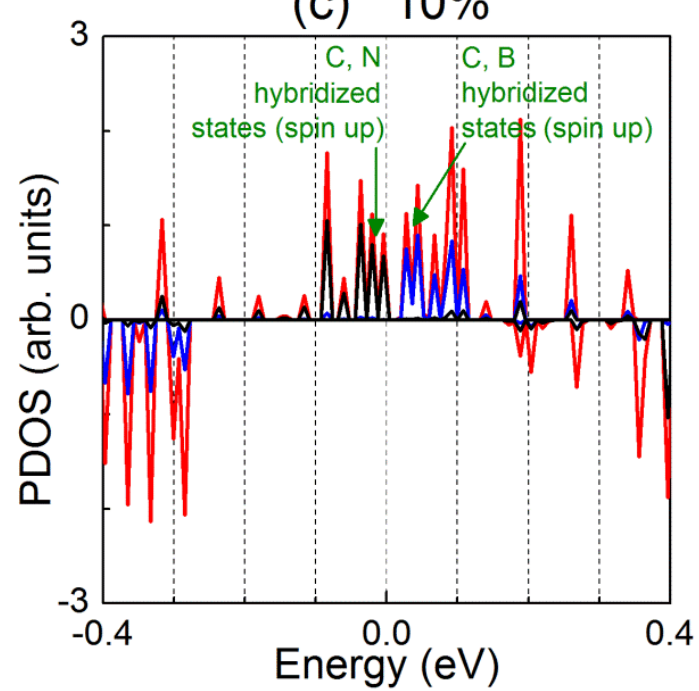

(b) $6 \%$

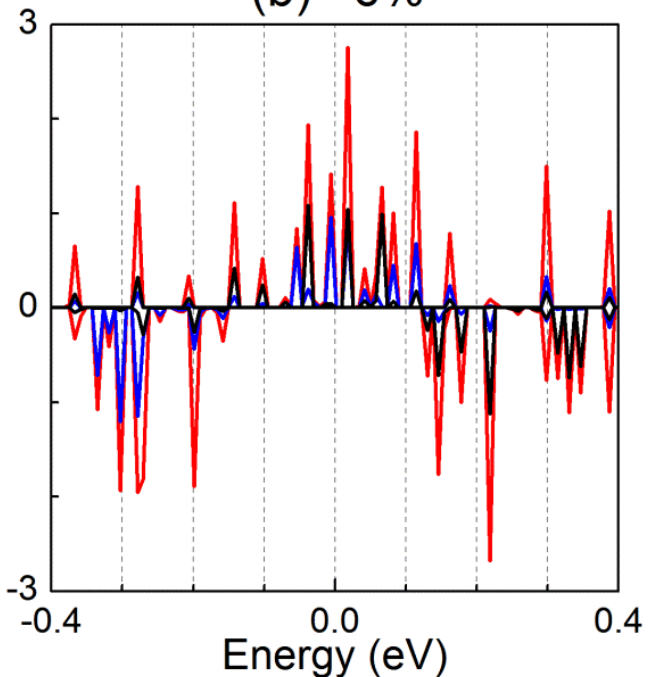

(d) $13 \%$

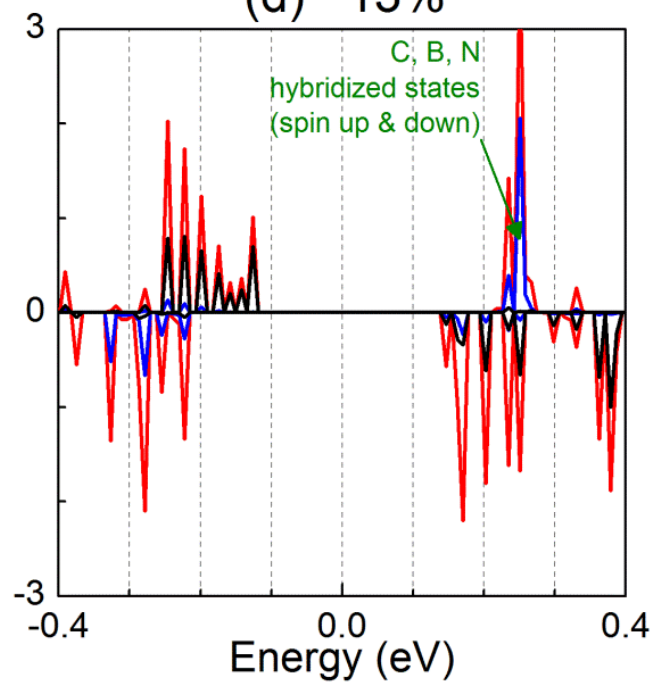

Figure 7. The projected density of states (PDOS) for $2 p$ orbitals as a function of tensile strain for zCBNSL10.

The different bandstructure and bandgap responses for spin-up and spin-down component are accompanied by specific variations of interfacial bond lengths (i.e., $\mathrm{C}-\mathrm{N}$ and $\mathrm{C}-\mathrm{B}$ ) under tension coupled with the Poisson effect. As shown in Fig. 8, the length of the C-B bond quickly increases with increasing strain, while the C-N bond length increases much slower. These bond length changes and the corresponding bandgaps in spin-up and spin-down components are in excellent agreement with the work reported in Ref. [27]. In addition, we note that similar strain- 
dependences of the spin-dependent band gaps are observed for all superlattice widths considered in this study (hence the use of zCBNSL10 as a representative in Fig. 8), thereby confirming that the interfacial bond lengths play an important role in bandgap variations of zCBNSL.

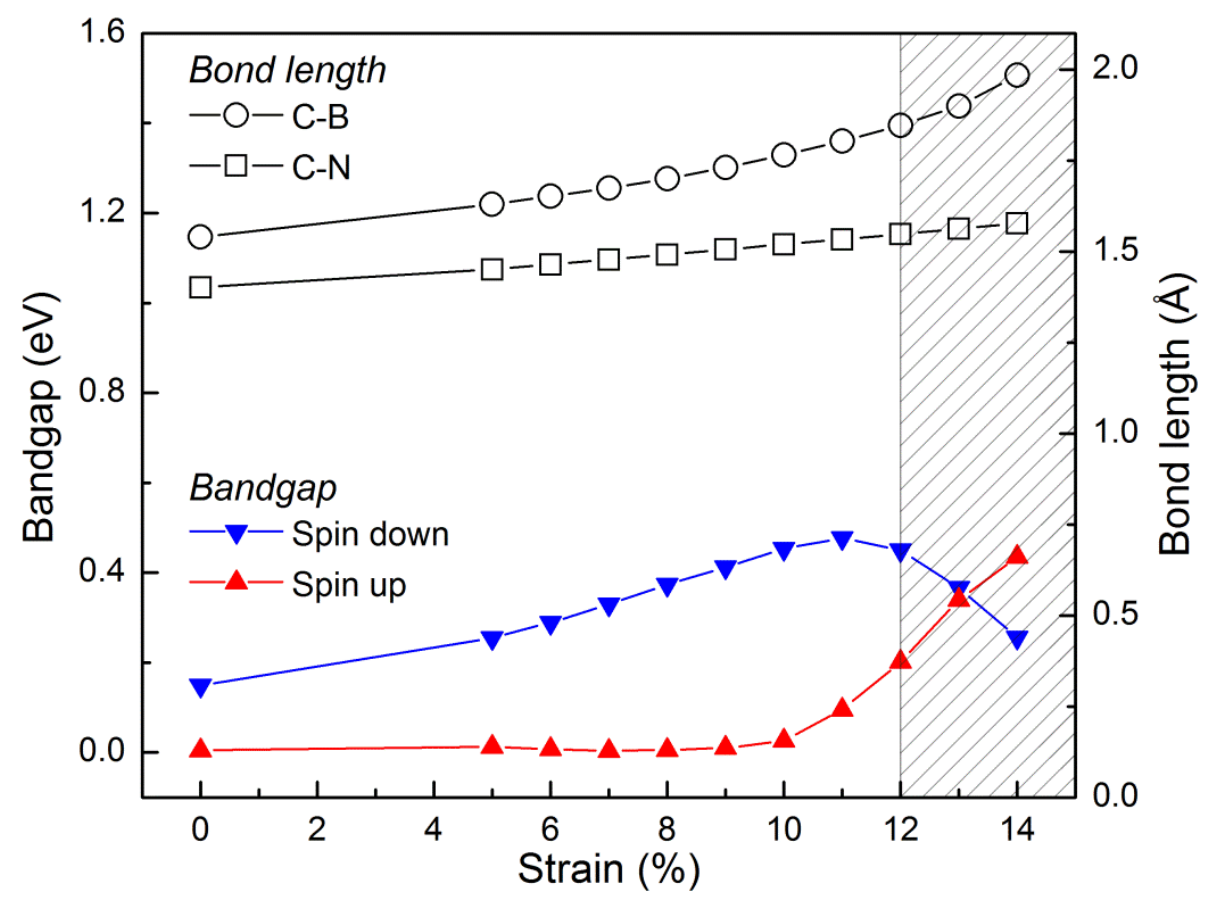

Figure 8. Bandgaps and interfacial bond lengths as a function of tensile strain for zCBNSL10.

As a final note, while we have pointed out the correlation between the strain-dependence of state mixing (hybridization shown in Fig. 7) and the spin-up and spin-down bandstructures, it is clear that further studies are necessary in order to form a complete understanding of the effect of strain on spintronic properties. The phenomenological underpinnings of this understanding are more likely to come from tight-binding modeling in which the analysis would be based on a small number of localized and well-known orbitals per atom and their interactions; such a study is outside of the scope of the DFT studies presented here.

Conclusion. In conclusion, our DFT calculations for monolayer hybrid domain CBNSLs have shown that: (i) for armchair CBNSLs under perpendicular strains, the total width acts as an 
effective one-parameter control of the bandgap value; the bandgap varies as an oscillatory function of the effective supercell widths; (ii) for aCBNSLs, strain can still be used as a way to control the bandgap value, and, independently, the domain width; (iii) for zCBNSLs, we have found that strain perpendicular to the domain boundary and the domain width independently change the bandstructure of each spin component. While most zCBNSLs are half-metallic at zero strain, the application of strain leads to a rich set of electronic and spintronic properties with strain beyond half-metallicity, including semiconductor behavior in each component, semiconductor behavior in one spin component and semimetal in the other, and reversal of halfmetallicity. These findings on the effect of strain and geometry on the spin-dependent bandstructure may give rise in the near future to applications in which strain is used as a way to control or actuate devices based on zCBNSL.

Acknowledgements. The authors gratefully acknowledge support from NSF through Grant No. CMMI-0856250 and the Ministry of Education, Science and Technology of Korea through Grant No. 2011K006.

\section{References}

[1] K.S. Novoselov, A.K. Geim, S.V. Morozov, D. Jiang, Y. Zhang, S.V. Dubonos, I.V. Grigorieva, A.A. Firsov, Electric field effect in atomically thin carbon films, Science, 306 (2004) 666-669.

[2] M.I. Katsnelson, K.S. Novoselov, A.K. Geim, Chiral tunnelling and the Klein paradox in graphene, Nat. Phys., 2 (2006) 620-625.

[3] S.V. Morozov, K.S. Novoselov, F. Schedin, D. Jiang, A.A. Firsov, A.K. Geim, Two-dimensional electron and hole gases at the surface of graphite, Phys. Rev. B, 72 (2005) 201401.

[4] K.S. Novoselov, A.K. Geim, S.V. Morozov, D. Jiang, M.I. Katsnelson, I.V. Grigorieva, S.V. Dubonos, A.A. Firsov, Two-dimensional gas of massless Dirac fermions in graphene, Nature, 438 (2005) 197-200. 
[5] K.S. Novoselov, D. Jiang, F. Schedin, T.J. Booth, V.V. Khotkevich, S.V. Morozov, A.K. Geim, Twodimensional atomic crystals, Proc. Natl. Acad. Sci. U. S. A., 102 (2005) 10451-10453.

[6] K.S. Novoselov, E. McCann, S.V. Morozov, V.I. Fal'ko, M.I. Katsnelson, U. Zeitler, D. Jiang, F. Schedin, A.K. Geim, Unconventional quantum Hall effect and Berry's phase of 2 pi in bilayer graphene, Nat. Phys., 2 (2006) 177-180.

[7] S.V. Morozov, K.S. Novoselov, M.I. Katsnelson, F. Schedin, D.C. Elias, J.A. Jaszczak, A.K. Geim, Giant intrinsic carrier mobilities in graphene and its bilayer, Physical Review Letters, 100 (2008) 016602.

[8] C. Lee, X.D. Wei, J.W. Kysar, J. Hone, Measurement of the elastic properties and intrinsic strength of monolayer graphene, Science, 321 (2008) 385-388.

[9] J.S. Bunch, S.S. Verbridge, J.S. Alden, A.M. van der Zande, J.M. Parpia, H.G. Craighead, P.L. McEuen, Impermeable atomic membranes from graphene sheets, Nano Letters, 8 (2008) 2458-2462.

[10] X.S. Li, W.W. Cai, J.H. An, S. Kim, J. Nah, D.X. Yang, R. Piner, A. Velamakanni, I. Jung, E. Tutuc, S.K. Banerjee, L. Colombo, R.S. Ruoff, Large-Area Synthesis of High-Quality and Uniform Graphene Films on Copper Foils, Science, 324 (2009) 1312-1314.

[11] S. Bae, H. Kim, Y. Lee, X.F. Xu, J.S. Park, Y. Zheng, J. Balakrishnan, T. Lei, H.R. Kim, Y.I. Song, Y.J. Kim, K.S. Kim, B. Ozyilmaz, J.H. Ahn, B.H. Hong, S. Iijima, Roll-to-roll production of 30-inch graphene films for transparent electrodes, Nature Nanotechnology, 5 (2010) 574-578.

[12] M.P. Levendorf, C.J. Kim, L. Brown, P.Y. Huang, R.W. Havener, D.A. Muller, J. Park, Graphene and boron nitride lateral heterostructures for atomically thin circuitry, Nature, 488 (2012) 627-632.

[13] Y.J. Kim, J.H. Lee, G.C. Yi, Vertically aligned ZnO nanostructures grown on graphene layers, Appl. Phys. Lett., 95 (2009) 213101.

[14] Z. Liu, L.L. Ma, G. Shi, W. Zhou, Y.J. Gong, S.D. Lei, X.B. Yang, J.N. Zhang, J.J. Yu, K.P. 
Hackenberg, A. Babakhani, J.C. Idrobo, R. Vajtai, J. Lou, P.M. Ajayan, In-plane heterostructures of graphene and hexagonal boron nitride with controlled domain sizes, Nature Nanotechnology, 8 (2013) 119-124.

[15] L. Ci, L. Song, C.H. Jin, D. Jariwala, D.X. Wu, Y.J. Li, A. Srivastava, Z.F. Wang, K. Storr, L. Balicas, F. Liu, P.M. Ajayan, Atomic layers of hybridized boron nitride and graphene domains, Nat. Mater., 9 (2010) 430-435.

[16] P. Sutter, R. Cortes, J. Lahiri, E. Sutter, Interface Formation in Monolayer Graphene-Boron Nitride Heterostructures, Nano Letters, 12 (2012) 4869-4874.

[17] Y. Gao, Y. Zhang, P. Chen, Y. Li, M. Liu, T. Gao, D. Ma, Y. Chen, Z. Cheng, X. Qju, W. Duan, Z. Liu, Toward Single-Layer Uniform Hexagonal Boron Nitride-Graphene Patchworks with Zigzag Linking Edges, Nano Letters, 13 (2013) 3439-3443.

[18] A.K. Geim, I.V. Grigorieva, Van der Waals heterostructures, Nature, 499 (2013) 419-425.

[19] Z. Liu, L. Song, S.Z. Zhao, J.Q. Huang, L.L. Ma, J.N. Zhang, J. Lou, P.M. Ajayan, Direct Growth of Graphene/Hexagonal Boron Nitride Stacked Layers, Nano Letters, 11 (2011) 2032-2037.

[20] R. Cortes, D.P. Acharya, C.V. Ciobanu, E. Sutter, P. Sutter, Graphene on Ru(0001) moire corrugation studied by STM on Au/graphene/Ru(0001) heterostructures, Journal of Physical Chemistry C, 117 (2013) 20675.

[21] A. Ebnonnasir, B. Narayanan, S. Kodambaka, C.V. Ciobanu, Tunable MoS2 bandgap in MoS2graphene heterostructures, Appl. Phys. Lett., 105 (2014) 031603.

[22] Y. Murata, S. Nie, A. Ebnonnasir, E. Starodub, B.B. Kappes, K.F. McCarty, C.V. Ciobanu, S. Kodambaka, Growth structure and work function of bilayer graphene on Pd(111) Phys. Rev. B, 85 (2012) 205443. 
[23] J. He, K.-Q. Chen, Z.-Q. Fan, L.-M. Tang, W.P. Hu, Transition from insulator to metal induced by hybridized connection of graphene and boron nitride nanoribbons, Appl. Phys. Lett., 97 (2010) 193305.

[24] S. Jun, X. Li, F. Meng, C.V. Ciobanu, Elastic properties of edges in BN and SiC nanoribbons and of boundaries in C-BN superlattices: A density functional theory study, Phys. Rev. B, 83 (2011) 153407.

[25] A. Ramasubramaniam, D. Naveh, Carrier-induced antiferromagnet of graphene islands embedded in hexagonal boron nitride, Phys. Rev. B, 84 (2011) 075405.

[26] J.-W. Jiang, J.-S. Wang, B.-S. Wang, Minimum thermal conductance in graphene and boron nitride superlattice, Appl. Phys. Lett., 99 (2011) 043109.

[27] J.M. Pruneda, Origin of half-semimetallicity induced at interfaces of C-BN heterostructures, Phys. Rev. B, 81 (2010) 161409.

[28] A. Ohtomo, H.Y. Hwang, A high-mobility electron gas at the LaAlO3/SrTiO3 heterointerface, Nature, 427 (2004) 423-426.

[29] Y.-W. Son, M.L. Cohen, S.G. Louie, Half-metallic graphene nanoribbons, Nature, 444 (2006) 347349.

[30] R. Martinez-Gordillo, M. Pruneda, Electromechanical response at polar zigzag boundaries in hybrid monolayers, Phys. Rev. B, 91 (2015) 045411.

[31] F. Liu, P. Ming, J. Li, Ab initio calculation of ideal strength and phonon instability of graphene under tension, Phys. Rev. B, 76 (2007) 064120.

[32] H. Zhao, K. Min, N.R. Aluru, Size and Chirality Dependent Elastic Properties of Graphene Nanoribbons under Uniaxial Tension, Nano Letters, 9 (2009) 3012-3015.

[33] Y. Zheng, N. Wei, Z. Fan, L. Xu, Z. Huang, Mechanical properties of grafold: a demonstration of 
strengthened graphene, Nanotechnology, 22 (2011) 405701.

[34] Q. Lu, W. Gao, R. Huang, Atomistic simulation and continuum modeling of graphene nanoribbons under uniaxial tension, Modell. Simul. Mater. Sci. Eng., 19 (2011) 054006.

[35] Y.Y. Zhang, Q.X. Pei, C.M. Wang, Mechanical properties of graphynes under tension: A molecular dynamics study, Appl. Phys. Lett., 101 (2012) 081909.

[36] X. Wei, B. Fragneaud, C.A. Marianetti, J.W. Kysar, Nonlinear elastic behavior of graphene: Ab initio calculations to continuum description, Phys. Rev. B, 80 (2009) 205407.

[37] J.M. Soler, E. Artacho, J.D. Gale, A. Garcia, J. Junquera, P. Ordejon, D. Sanchez-Portal, The SIESTA method for ab initio order-N materials simulation, Journal of Physics-Condensed Matter, 14 (2002) 27452779.

[38] N. Troullier, J.L. Martins, EFFICIENT PSEUDOPOTENTIALS FOR PLANE-WAVE

CALCULATIONS, Phys. Rev. B, 43 (1991) 1993-2006.

[39] J.P. Perdew, K. Burke, M. Ernzerhof, Generalized gradient approximation made simple, Physical Review Letters, 77 (1996) 3865-3868.

[40] S. Reich, J. Maultzsch, C. Thomsen, P. Ordejon, Tight-binding description of graphene, Phys. Rev. B, 66 (2002) 035412.

[41] Y.-W. Son, M.L. Cohen, S.G. Louie, Energy gaps in graphene nanoribbons, Physical Review Letters, 97 (2006) 216803. 

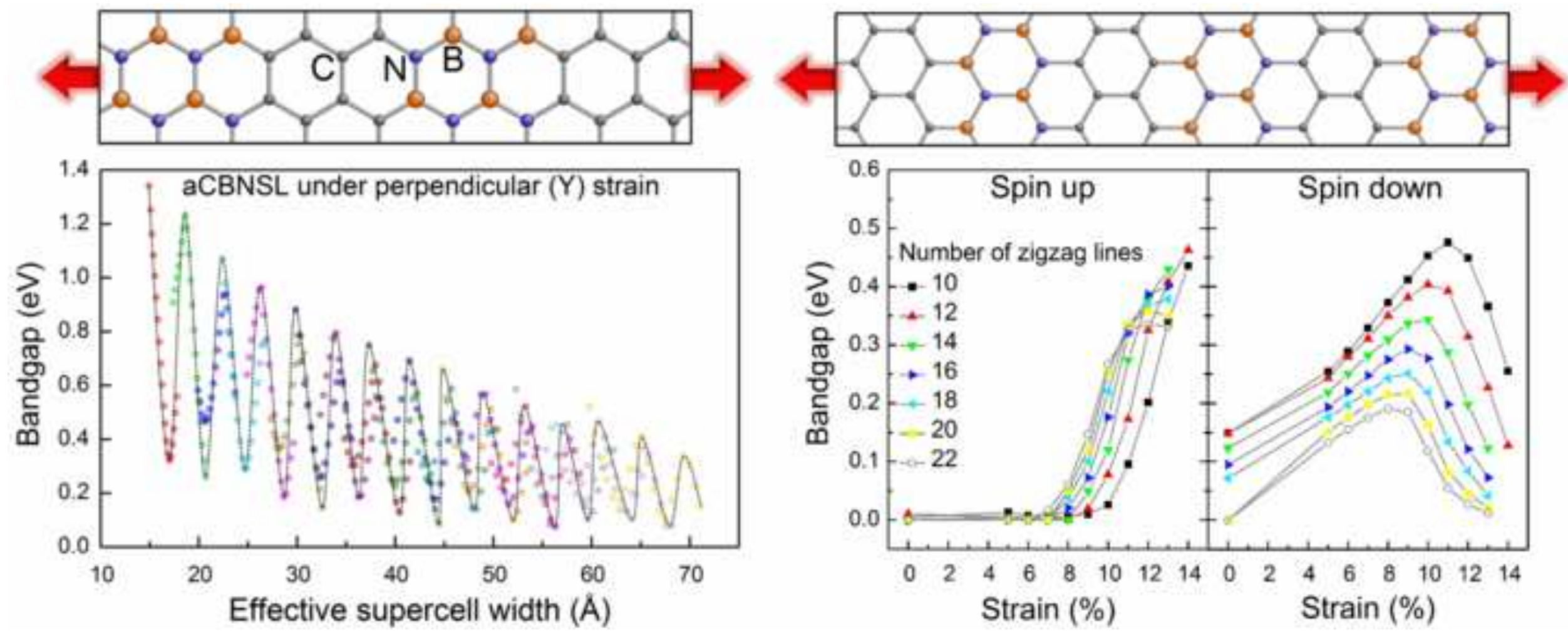